\title{
Acting locally: innate mucosal immunity in resistance to HIV-1 infection in Kenyan commercial sex workers
}

\author{
X-D Yao ${ }^{1}$, RW Omange $^{2}$, BM Henrick ${ }^{1}$, RT Lester ${ }^{3}$, J Kimani ${ }^{4}$, TB Ball ${ }^{2}$, FA Plummer ${ }^{2}$ and KL Rosenthal $^{1}$
}

Cohort studies of female commercial sex workers (CSWs) in Kenya were among the first to identify highly HIV-1-exposed seronegative (HESN) individuals. As natural resistance is usually mediated by innate immune mechanisms, we focused on determining whether expression and function of innate signaling pathways were altered locally in the genital mucosa of HESN CSWs. Our results demonstrated that selected pattern-recognition receptors (PRRs) were significantly reduced in expression in cervical mononuclear cells (CMCs) from HESN compared with the new HIV-negative (HIV-N) and HIV-positive (HIV-P) groups. Although baseline levels of secreted cytokines were reduced in CMCs of HESN, they were highly stimulated following exposure to ssRNA40 in vitro. Importantly, cervical epithelial cells from HESN also expressed reduced levels of PRRs, but Toll-like receptor 3 (TLR3) and TLR7 as well as nuclear factor- $\mathrm{KB}$ and activator protein 1 were highly expressed and activated. Lastly, inflammatory cytokines interleukin (IL)-1 $\beta$, IL-8, and RANTES (regulated and normal T cell expressed and secreted) were detected at lower levels in cervicovaginal lavage of HESN compared with the HIV-N and HIV-P groups. Overall, our study reveals a local microenvironment of HIV resistance in the genital mucosa consisting of a finely controlled balance of basal immune quiescence with a focused and potent innate anti-viral response critical to resistance to sexual transmission of HIV-1.

\section{INTRODUCTION}

Cohort studies of commercial sex workers (CSWs) in Nairobi, Kenya were among the first to identify a subgroup of women who were highly exposed to HIV-1 for many years but remained uninfected. ${ }^{1}$ Such highly HIV-1-exposed seronegative (HESN) populations have also been identified among discordant couples, ${ }^{2}$ intravenous drug users, ${ }^{3}$ and children born to HIV-infected mothers. ${ }^{4,5}$ Studies of HESN individuals have revealed a number of correlates associated with HIV resistance, including genetic, viral, immunological, and sociobehavioral factors. Nevertheless, the precise mechanisms of protection against HIV in such individuals remain to be determined.

Early studies in HESN CSWs in Nairobi indicated that local mucosal factors in the cervicovaginal compartment had a key role in resistance. ${ }^{6,7}$ Indeed, it was shown that HESN woman had specific anti-HIV-1 immunoglobulin A in genital washes compared with HIV-negative (HIV-N). HESN CSWs have also been shown to have significantly elevated $\mathrm{T}$-cell counts $(\mathrm{CD} 3+, \mathrm{CD} 4+$, and $\mathrm{CD} 8+)$ in the genital tract that specifically responded to HIV-1 peptides in vitro compared with either uninfected susceptible CSWs (HIV-N) or infected women (HIV-positive (HIV-P)). ${ }^{8}$ Further, chemokine-expressing patterns and HIV-1 coreceptor levels were altered in HESN CSWs. ${ }^{8,9}$ More recently, findings of lower basal T-cell gene expression and reduced T-cell activation in HESN peripheral blood mononuclear cells (PBMCs) led to the "immune quiescence" hypothesis of HIV resistance. ${ }^{10-12}$ Furthermore, screening of cervicovaginal lavage (CVL) identified trappin-2/ elafin as biomarkers of resistance in HESN ${ }^{13}$ and proteomic studies identified other antiproteases in HESN CVL. ${ }^{14}$

Natural resistance to most infections is mediated by the innate immune system. Pattern-recognition receptors (PRRs)

${ }^{1}$ McMaster Immunology Research Centre, Michael G. DeGroote Institute for Infectious Disease Research, Department of Pathology and Molecular Medicine, McMaster University, Hamilton, Ontario, Canada. ${ }^{2}$ Univeristy of Manitoba and Public Health Agency of Canada, Winnipeg, Manitoba, Canada. ${ }^{3}$ BCCDC, University of British Columbia, Vancouver, British Columbia, Canada and ${ }^{4}$ Department of Medical Microbiology, University of Nairobi, Nairobi, Kenya. Correspondence: KL Rosenthal (rosenthl@mcmaster.ca)

Received 4 March 2013; accepted 20 May 2013; published online 26 June 2013. doi:10.1038/mi.2013.44 
have a central role in initiating innate immune activation and bridging the gap to adaptive immunity. To date, numerous PRRs have been identified in humans, including Toll-like receptors (TLRs), retinoic acid-inducible gene-1 (RIG-I)-like receptors (RLRs), C-type lectin receptors, Nod-like receptors, and DNA-dependent activator of interferon (IFN)-regulatory factors, a cytosolic DNA sensor. These receptors are part of first-line molecules critical to host defense against invading pathogenic microorganisms and are also potent sensors to be activated for inflammatory reactions.

In recent years, increasing evidence supports the hypothesis that immune activation enhances HIV-1 infection and chronic disease progression ${ }^{15}$ and that TLR-pathogen interactions may have an indirect role in regulating HIV-associated disease. ${ }^{16}$ In addition, HIV itself may directly induce immune activation and dysfunction by stimulating TLR7, thereby contributing to progressive immunodeficiency. ${ }^{17-19}$ In a previous study, we showed that chronic, untreated HIV-1 infection was significantly associated with increased mRNA expression of TLR6, TLR7, and TLR8 in PBMCs, and in advanced disease states TLR2, TLR3, and TLR4 were additionally elevated. ${ }^{20}$ Further, expression of TLR6 and TLR7 was significantly correlated with plasma HIV-RNA load. Importantly, TLR expression was normalized following treatment with HAART (highly active antiretroviral therapy). ${ }^{20}$ PBMCs of HIV-infected subjects demonstrated profoundly increased pro-inflammatory responses to TLR ligands, suggesting sensitization of TLR signaling in HIV. Therefore, chronic untreated HIV-1 infection was associated with increased TLR expression and responsiveness, which may perpetuate innate immune activation and dysfunction. ${ }^{9}$

Genetic mechanisms, e.g., CCR $5 \Delta 32$, do not fully account for resistance to HIV-1 infection in Kenyan HESN CSWs. ${ }^{13,14}$ Therefore, HIV-1-specific protective immune responses may exist that help to explain this phenomenon. Our more recent observations reveal that HESN CSWs contain significantly higher amounts of serine antiprotease trappin-2 (Tr) and its processed form, elafin (E), in CVL fluid, compared with HIV-N CSWs. ${ }^{21}$ Analysis of the cytokines in the serum of HESN women revealed hypo-expression of cytokine interleukin (IL)- $4 .^{22}$

The genital mucosa is a point of vulnerability as well as a location that is crucial for the success of HIV-1 in transmission and establishing a persistent infection. ${ }^{23}$ In this study, we assessed innate immune PRR expression and responses in different cellular compartments of the genital mucosa of Kenyan female HESN compared with HIV-N and HIV-P CSWs. HESN CSWs were enrolled in our study cohort following $>7$ years in commercial sex work. HIV-N CSWs were newly enrolled seronegative CSWs with $<3$ years of sex work. These women were presumed to be susceptible to HIV-1 infection but had a shorter history in sex work. Clearly, we cannot exclude the possibility that some of the HIV-N may become HESN with time in sex work. We also utilized historic specimens from a HESN cohort from 2005. At that time, women who were engaged in sex work for $>3$ years were considered HESN. We hypothesize that innate immunity in the local genital microenvironment will display differences between HESN, HIV-N, and HIV-P CSWs. Overall, we identified a unique mucosal microenvironment that favors local HIV resistance in the female genital tract, and this environment involves immune quiescence with a potent antiviral response.

\section{RESULTS \\ Significantly reduced expression of selected PRRs in HESN cervical mononuclear cells (CMCs)}

Initially, we attempted to assess the expression of various PRRs, including TLRs, and RLRs, including RIG-I, and melanomadifferentiation-associated gene 5 (Mda-5). CMCs were collected and isolated from the ectocervix and endocervix of the CSWs, and the purified RNA samples were quantified using quantitative reverse transcriptase-PCR. The mRNA expression of TLR2, RIG-I, and Mda-5 was significantly reduced in CMCs of the HESN group compared with the HIV-N group and highly significantly reduced expression compared with the HIV-P group (Figure 1a). Similarly, UNC93B, a protein involved in intracellular trafficking of TLRs from the endoplasmic reticulum to endosomes, ${ }^{24}$ was also expressed at highly significantly decreased levels in CMCs of HESN compared with the HIV-N and HIV-P groups. Although no significant differences between HESN and HIV-N were observed in the mRNA expression levels of TLR4 and TLR6 and the downstream signaling genes Janus-activated kinase 2 (JAK2) and IFN $\gamma$, these genes were expressed at significantly lower levels in HESN compared with HIV-P (Figure 1b). TLR6 and JAK2 were also expressed at significantly reduced levels in HIV-N compared with the HIV-P group. Overall, these data indicate that selected PRRs involved in innate sensing and signaling pathways were significantly reduced in expression in CMCs from HESN compared with HIV-N and HIV-infected CSWs.

\section{HESN women maintain decreased TLR7 and TLR8 expression in CMCs over time}

Although the data shown above was determined using CMCs collected at one time point, from the 35th resurvey of the CSW cohort in 2009, CMCs from this cohort had been collected by our group since 2005. Here, we compared the mRNA expression of TLR7 and TLR8 in CMCs from 2009 (Figure 2a), as well as historic CMC specimens from 2007 (Figure 2b) and 2005 (Figure 2c). These studies were conducted separately. In 2005, women were considered HESN if they were engaged in commercial sex work for $>3$ years and remained uninfected. At this time, there was a high prevalence of HIV-1 in the population. ${ }^{1}$ Whereas in 2007 and 2009, women engaged in sex work for $>7$ years without infection were considered HESN. The definition of HIV-N remained the same, $<3$ years in CSW while remaining HIV-1 seronegative. Data from 2005 and 2007 assessments showed significantly or a trend of reduced expression of TLR7 and TLR8 in CMCs of HESN compared with the HIV-N or HIV-P group (Figure 2b,c). Results (Figure 2a) from 2009 showed that TLR8 was expressed at significantly reduced levels compared 
a
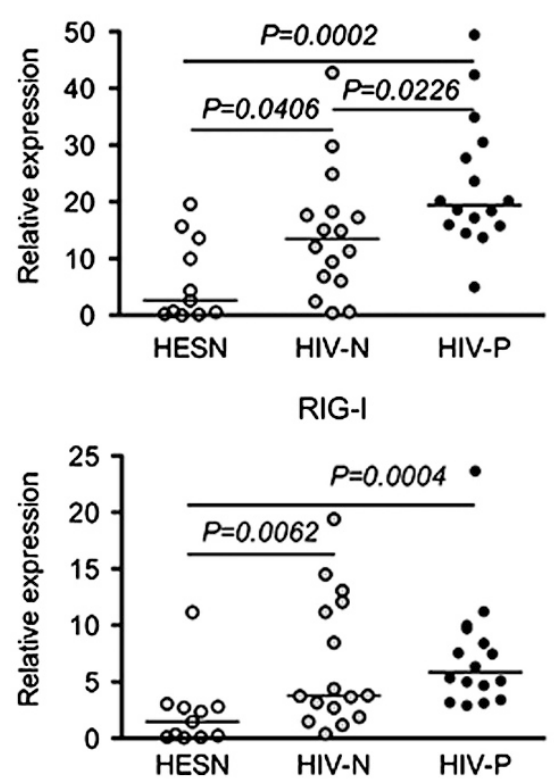

b

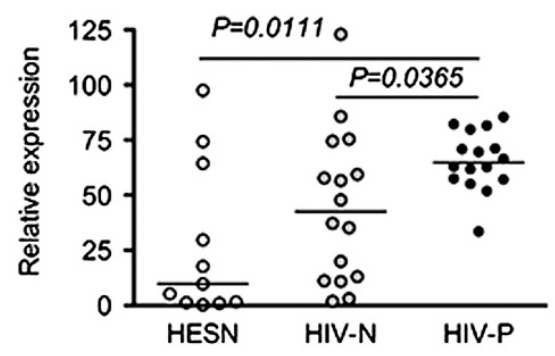

IFN $\gamma$

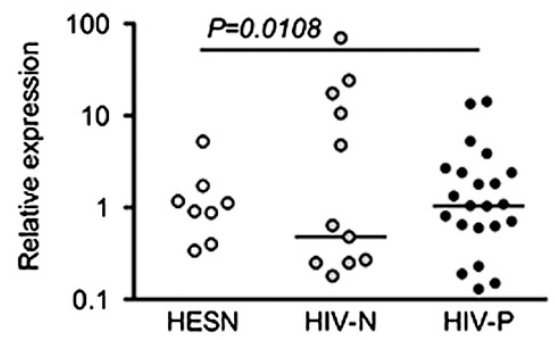

UNC93B
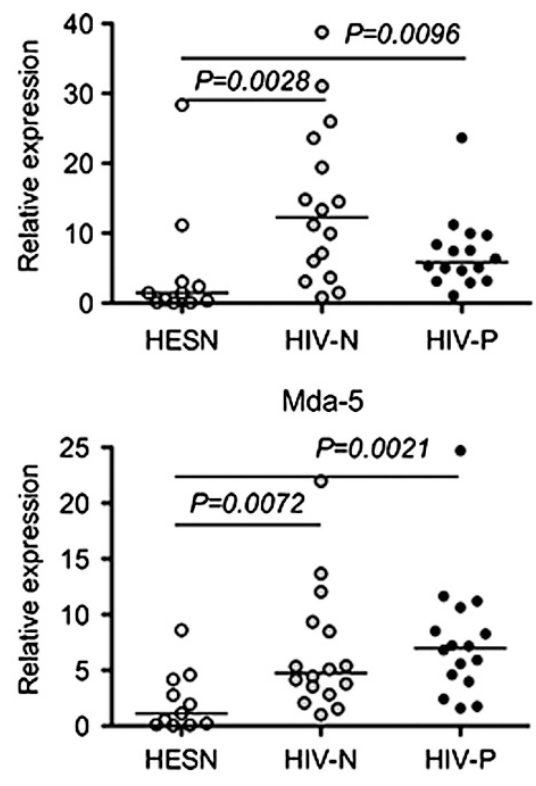

TLR6

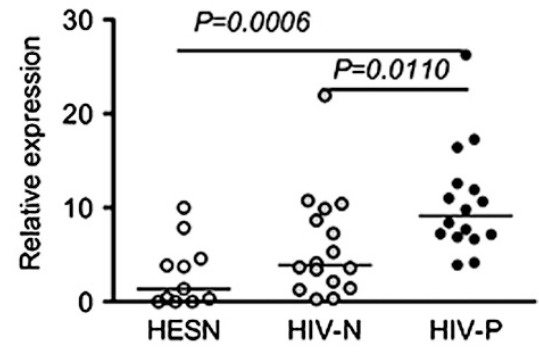

JAK2

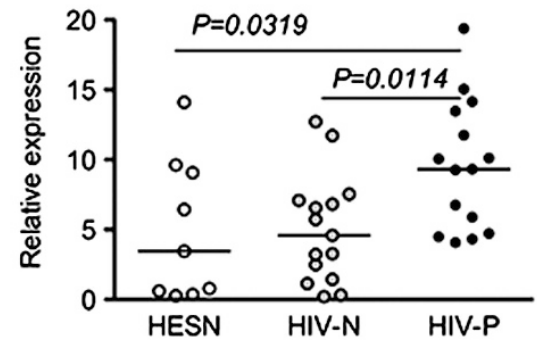

Figure 1 Significantly reduced expression of selected pattern-recognition receptors in cervical mononuclear cells of HIV-1-exposed seronegative (HESN). (a) HESN samples had significantly decreased mRNA expression of Toll-like receptor 2 (TLR2), UNC93B, retinoic acid-inducible gene-1 (RIG-I), and melanoma-differentiation-associated gene 5 (Mda-5) compared with HIV-negative (HIV-N), and very significantly decreased levels compared with HIV-positive (HIV-P). (b) Significantly decreased mRNA expression of TLR4, TLR6, TLR8, interferon- $\gamma$ (IFN $\gamma$ ), and Janus-activated kinase 2 (JAK2) were demonstrated in HESN vs. HIV-P. Relative expression was determined by normalizing detection quantity with $18 \mathrm{~S}$ rRNA.

Significantly statistic $P$-values $<0.05$ are shown in the plots.

with HIV-N and HIV-P, but expression of TLR7 was not significantly different. Overall, expression of TLR7 and TLR8 in the various groups maintained similar patterns over a number of years of assessment. These results support a previous finding that HIV replication requires TLR8-mediated signaling in dendritic cells. ${ }^{25}$

In downstream signaling pathways, IL-6 mRNA expression levels in CMCs sampled in 2005 were also significantly reduced in HESN compared with HIV-P (Supplementary Figure S1 online). Our finding of lower inflammatory IL-6 may be associated with lower levels of PRR expression and reflect reduced innate immune activation of genital CMCs from HESN CSWs. Indeed, functional studies of adaptive immune responses and genomic studies of the HESN CSWs have been reported to display an "immune quiescent" state. ${ }^{11,26}$

\section{Significantly increased response of CMCs from HESN CSWs to stimulation with HIV-1 ssRNA}

Observing markedly reduced expression of numerous PRRs in CMCs of HESN, we sought to determine whether these CMCs 
a

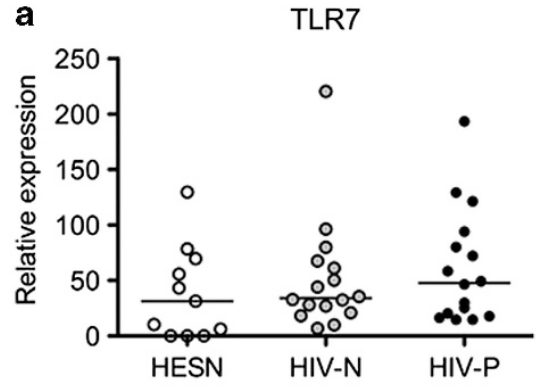

b
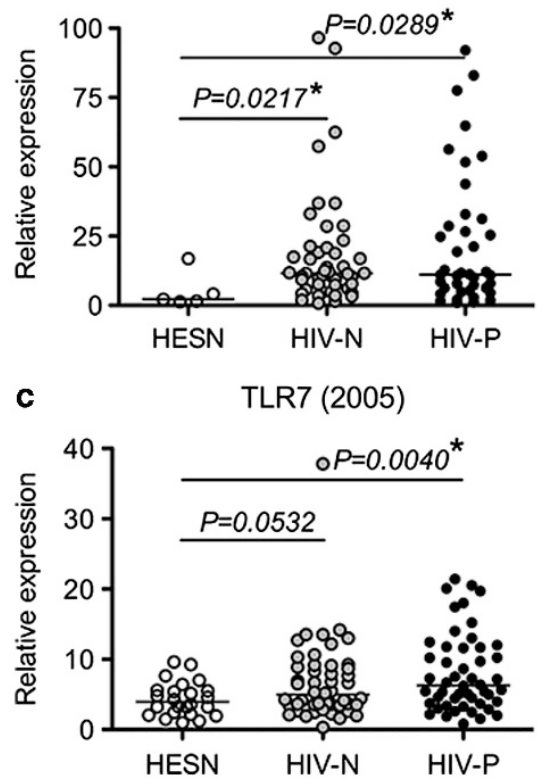
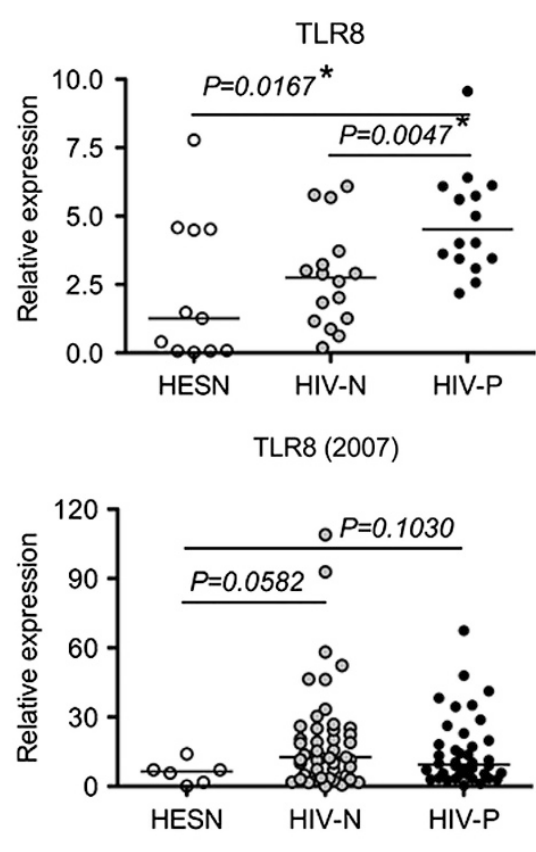

TLR8 (2005)

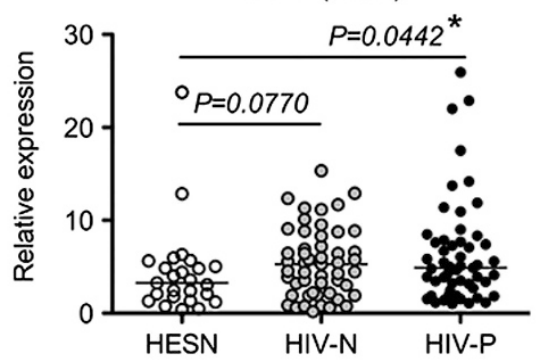

Figure 2 Reduced expression of Toll-like receptor 7 (TLR7) and TLR8 in cervical mononuclear cells (CMCs) of HIV-1-exposed seronegative (HESN) commercial sex workers (CSWs) collected in different years. (a) Relative levels of gene expression to 18S rRNA were measured in current CMC samples from year 2009. CSWs were considered HESN if they had been in sex work for $>7$ years. (b) CMC samples were from year 2005 and women in sex work for $>3$ years were considered HESN. Relative expression was normalized to 18S rRNA. (c) Data were from CMC samples collected in year 2007. CSWs who remained uninfected for $>7$ years were considered HESN. Relative expression was normalized to L32. $P$-values with less or around 0.05 are shown in the plots. HIV-N, HIV-negative; HIV-P, HIV-positive.

were dysfunctional and to compare the responsiveness of these cells with pathogen stimulation. CMCs are a group of immune cells, including mucosal macrophages, T cells, NK cells, B cells, and dendritic cells. We evaluated the overall cytokine production of these cells by culturing isolated CMCs and treating with TLR4 ligand, lipopolysaccharide (LPS), or TLR7/8 ligand, ssRNA40, or TLR7-specific ligand, imiquimod, using multiplex cytometric bead arrays. Of the six cytokines assessed, including IL-2, IL-4, IL-6, IL-10, TNF- $\alpha$, and IFN $\gamma$, only the levels of TNF- $\alpha$ and IL-10 in culture supernatants were significantly different. The basal levels of TNF- $\alpha$ and IL-10 secreted by CMC in vitro were significantly lower in HESN compared with the HIV-N group (Figure 3). By contrast, production of both cytokines was significantly elevated in HESN compared with both the HIV-N and HIV-P groups following exposure of CMCs to ssRNA40 (Figure 3). However, production of the cytokines by CMCs in the presence of LPS and imiquimod was detected to similar extent among all the groups. The lower basal levels of cytokine production in CMCs of HESN women provide additional evidence supporting the concept that a lower state of immune activation or quiescent phenotype of immune cells may be linked with preventing inflammation locally in genital mucosa and protection of this group of women against HIV infection. Notably, though, once in the presence of ssRNA40, a uridine-rich ssRNA that is analogous to HIV-1 ssRNA, CMCs of HESN actively responded to TLR7 and TLR8 stimulation by producing a greater amount of both pro- and anti-inflammatory cytokines.

\section{Significantly reduced levels of indoleamine-2,3- dioxygenase 1 (IDO1) in CMCs of HESN compared with HIV-infected CSWs}

The products of IDO, tryptophan catabolites including kynurenines, can induce the generation of FoxP3 + regulatory T cells and inhibit T helper type 17 (Th17) generation. ${ }^{27}$ More recent studies ${ }^{28}$ have shown that progressive HIV disease was associated with loss of Th17 cells and an increase in immunosuppressive regulatory $\mathrm{T}$ cells in blood and gut 

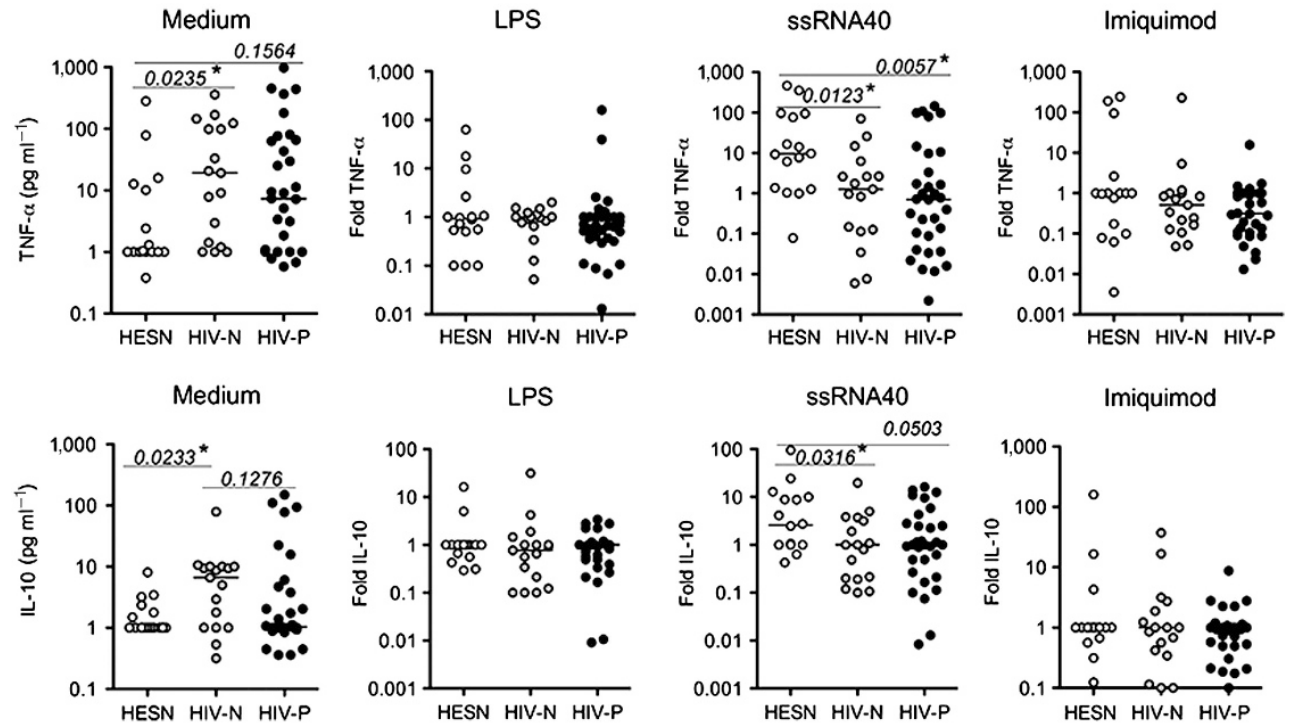

Figure 3 Reduced basal expression of cytokines and significantly increased responses to ssRNA40 by HIV-1-exposed seronegative (HESN) cervical mononuclear cells (CMCs) in vitro. Tumor necrosis factor (TNF)- $\alpha$ and interleukin (IL)-10 were measured in the supernatants of cultured CMCs. Cytokine concentration in CMCs treated with Toll-like receptor ligands, lipopolysaccharide (LPS), ssRNA40, and imiquimod were normalized to untreated CMCs. Concentrations of TNF- $\alpha$ and IL-10 $\left(\mathrm{pg} \mathrm{ml}^{-1}\right)$ were plotted in the untreated samples, and cytokine fold changes were plotted in the ligand-treated samples. ${ }^{*} P<0.05$. HIV-N, HIV-negative; HIV-P, HIV-positive.

mucosa, and this was associated with induction of IDO1. Chronic stimulation of IDO1 in HIV infection would lead to increased activation of the tryptophan oxidation pathway, disrupted mucosal integrity with increased microbial translocation, and chronic inflammation. Indeed in contrast to pathogenic simian immunodeficiency virus (SIV) infection, IDO1 activity returned to baseline levels in non-pathogenic SIV infection. ${ }^{29}$ In support of these findings, our data confirmed significantly higher levels of IDO1 expressed in genital CMCs of HIV-P. Importantly, IDO1 expression was significantly reduced in CMCs of HESN CSWs (Figure 4), which might reflect more stable genital mucosa.

\section{Cervical epithelial cells (CECs) from HESN express significantly higher levels of TLR3 and TLR7}

Next we isolated CECs from the CSWs and compared the mRNA expression levels of various PRRs in the three groups of women. As shown in Figure 5a, mRNA expression levels of TLR2, TLR4, and UNC93B were significantly decreased in CECs of HESN compared with both the HIV-N and HIV-P groups. Mda-5 also showed significantly reduced expression in CECs of HESN compared with HIV-P. TLR6 and RIG-I expression in HESN CECs showed a trend of lower mRNA levels compared with the other two groups. The reduced expression of most PRRs in CECs was similar to the expression pattern previously observed in CMCs from HESN. Importantly, expression of TLR3 and TLR7 were remarkably enhanced in CECs from HESN compared with HIV-P, as well as a trend of higher levels in HESN vs. HIV-N (TLR3: $P=0.0643$, TLR7: $P=0.0868$ ), although quantitative values varied widely among the samples (Figure 5a). Though not significant, TLR8 in CECs displayed a similar expression pattern to TLR3 and TLR7. Correlation analysis showed that TLR3 levels correlated very strongly to

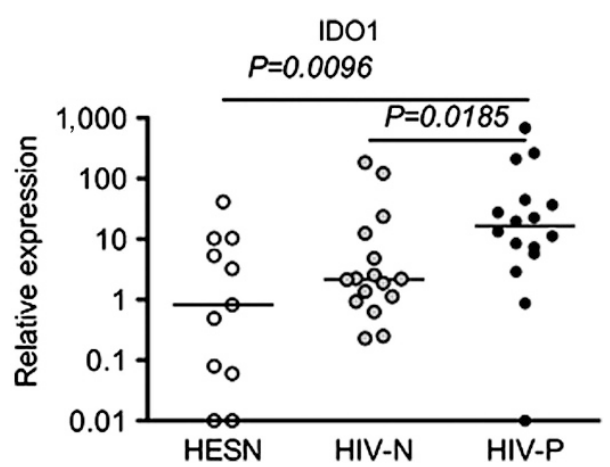

Figure 4 Significantly increased indoleamine-2,3-dioxygenase 1 (IDO1) expression in cervical mononuclear cells (CMCs) of HIV-P compared with HIV-1-exposed seronegative (HESN) and HIV-negative (HIV-N) groups. Relative expression of IDO1 was determined in CMCs from the three groups and normalized to $18 \mathrm{~S}$ rRNA with quantitative reverse transcriptase-PCR. Significant $P$-values $(<0.05)$ are indicated on the plot. HIV-P, HIV-positive.

TLR7 levels in all the three groups (Figure 5b), while TLR7 expression levels also significantly correlated to TLR8 in the HESN and HIV-P groups (Figure 5b). These results demonstrate that intracellularly located endosomal PRRs, TLR3, TLR7, and TLR8, specific for sensing dsRNA or ssRNA, were highly expressed in CECs, the first line of mucosal defense in the genital tract, and may be key factors in local resistance to infection in HESN CSWs.

Nuclear factor (NF)-кB and activator protein 1 (AP-1) are highly expressed and activated in CECs from HESN, while ISG15 is markedly reduced in expression

As PRRs converge on a few downstream transcription factors that serve as molecular hubs to connect multiple signaling pathways in different cells, we next attempted to examine which 
a
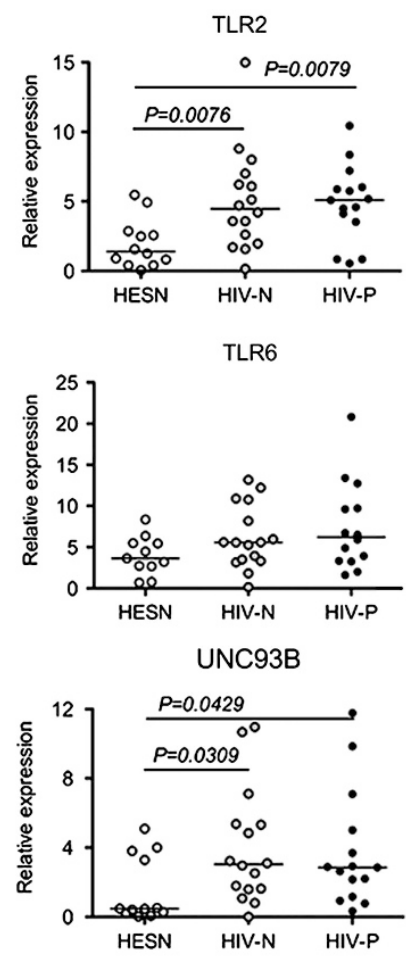

b

TLR3 vs. TLR7 in HESN

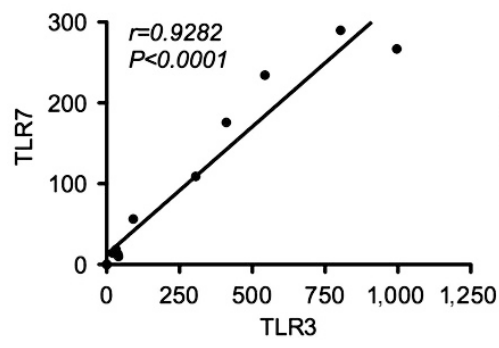

TLR7 vs. TLR8 in HESN

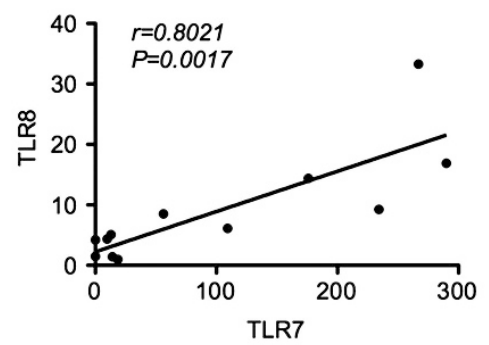

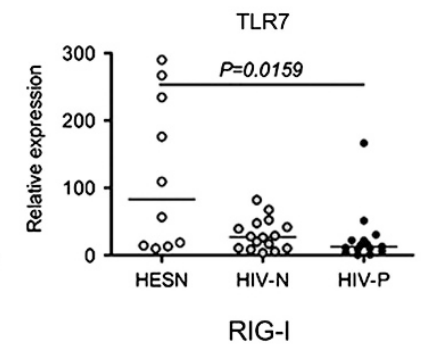
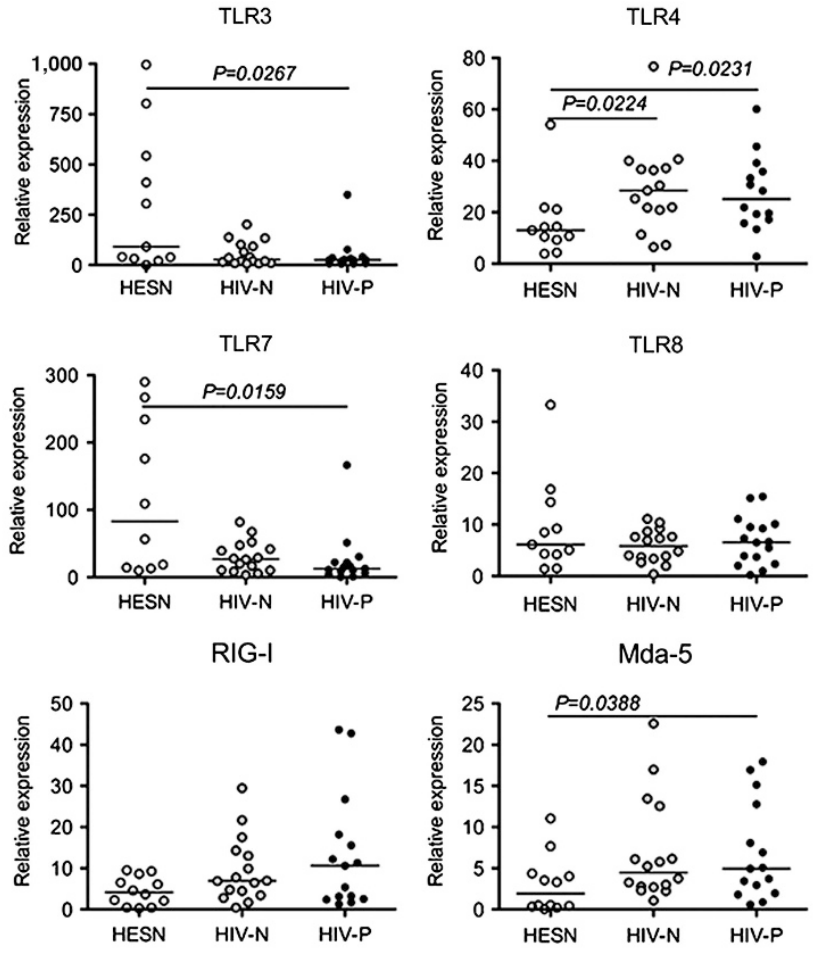

TLR3 vs. TLR7 in HIV-N

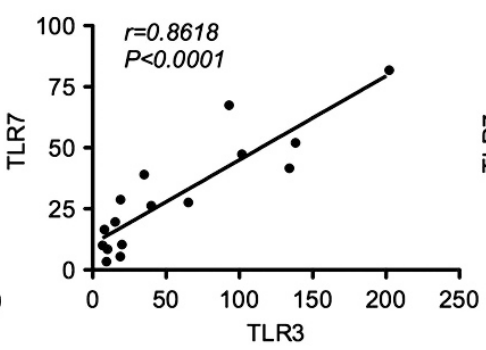

TLR7 vs. TLR8 in HIV-N

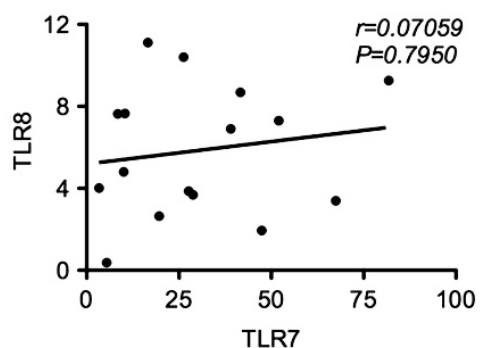

TLR3 vs. TLR7 in HIV-P

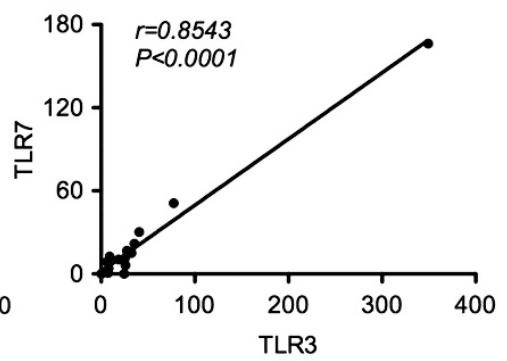

TLR7 vs. TLR8 in HIV-P

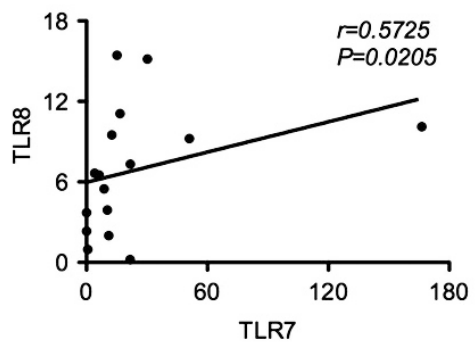

Figure 5 Differential expression and correlation of selected pattern-recognition receptors in cervical epithelial cells (CECs). (a) Toll-like receptor 2 (TLR2), TLR3, TLR6, TLR7, TLR8, UNC93B, retinoic acid-inducible gene-1 (RIG-I) and melanoma-differentiation-associated gene 5 (Mda-5) were measured with quantitative reverse transcriptase-PCR and relative quantity was normalized to $18 \mathrm{~S}$ rRNA. $P<0.05$ is shown.

(b) TLR3 expression positively correlated with TLR7 and TLR8 from HIV-1-exposed seronegative (HESN), HIV-negative (HIV-N), and HIV-positive (HIV-P) CECs. Expression of TLR7 and TLR8 was positively correlated in the HESN and HIV-P groups. Spearman $r$ and $P$-values are shown in the plots.

transcription factors were involved and activated. Expression levels of NF- $\kappa \mathrm{B}$ subunits $\mathrm{p} 65$ and phosphorylated inhibitor $\kappa \mathrm{B} \alpha(\mathrm{I} \kappa \mathrm{B} \alpha), \mathrm{AP}-1$ subunit $\mathrm{c}-\mathrm{Jun}$, and its active form, phosphorylated c-Jun, were detected in CECs using western blot. As shown in Figure 6a, the normalized quantity of p65 was greatly increased in CECs from the HESN group compared with the other two groups. Phosphorylation of another subunit of NF- $\kappa \mathrm{B}$, I $\mathrm{B} \alpha$, demonstrated higher levels in HESN and HIV-P compared with HIV-N, in which phosphorylation of serine $32 / 36$ is a strong marker of 
NF- $\kappa \mathrm{B}$ activation. ${ }^{30-32} \mathrm{c}$-Jun was also highly expressed in HESN and HIV-P compared with HIV-N, and active c-Jun showed the same patterns. Enhanced expression and activation of both NF- $\kappa \mathrm{B}$ and AP-1 might be relative to highly expressed TLR3 and TLR7 in HESN CECs. Figure $6 \mathbf{b}$ showed no obvious change of interferon regulatory factor 3 (IRF3) protein levels in CECs among the three groups, but ISG15, one of the IRF3regulated genes, was expressed at markedly lower levels in HESN compared with the HIV-N and HIV-P groups (Figure 6c).

\section{Inflammatory cytokine concentrations are reduced in CVL from HESN}

Inflammatory cytokines IL-1 $\beta$, IL8, RANTES (regulated and normal $\mathrm{T}$ cell expressed and secreted; i.e., C-C motif chemokine ligand 5 (CCL5)), and TNF- $\alpha$ were measured in CVL from all CSWs. Despite repeated attempts, TNF- $\alpha$ was too low to be detected. However, other inflammatory cytokines IL-1 $\beta$, IL-8, and RANTES were all detectable and indicated lower levels in CVL of HESN compared with the HIV-N and HIV-P groups (Figure 7). The lower levels of these inflammatory cytokines in
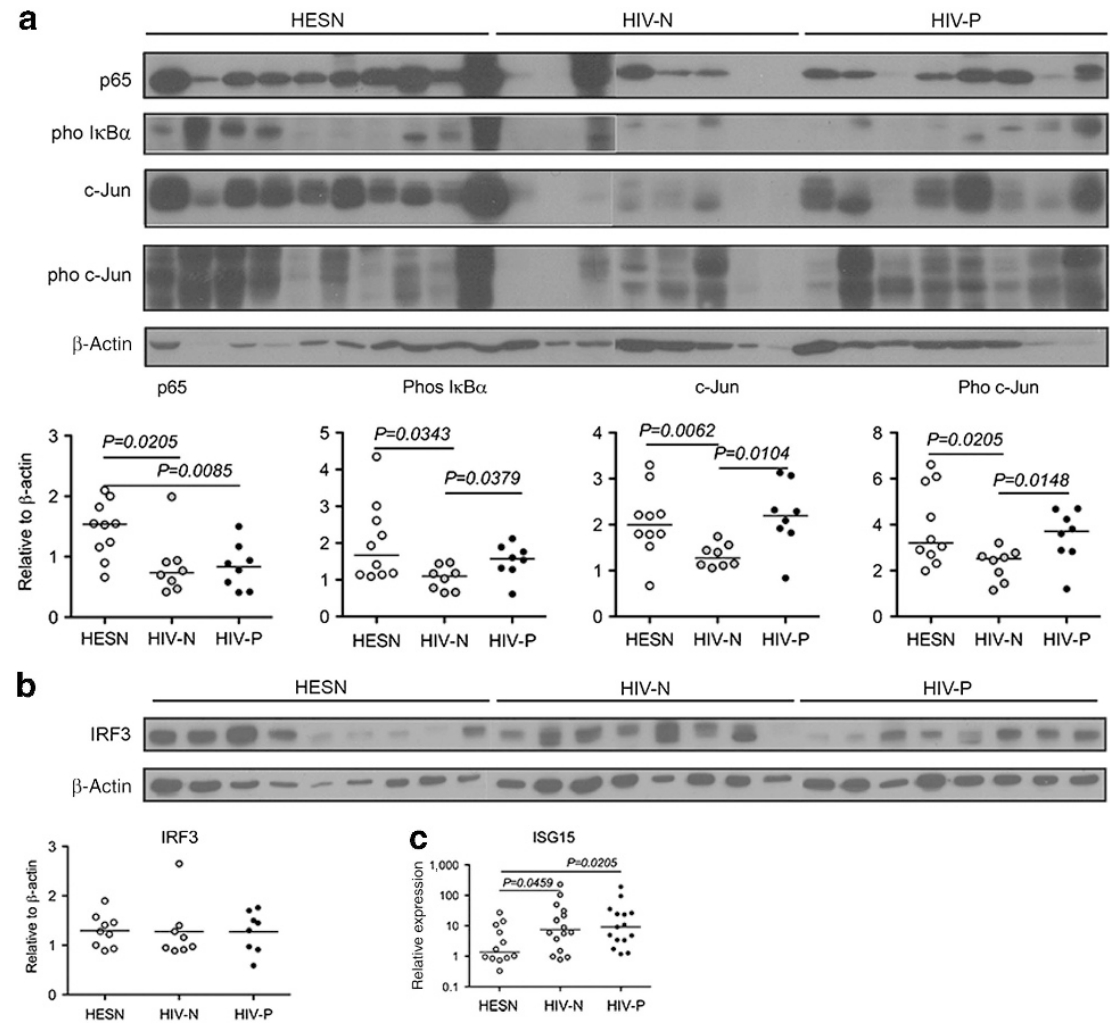

Figure 6 Increased expression and activation of transcription factors nuclear factor (NF)-KB and activator protein 1 (AP-1) in HIV-1-exposed

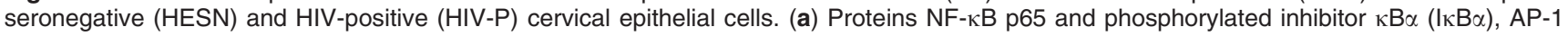
components c-Jun and phosphorylated c-Jun from cervical mononuclear cells were detected with SDS-10\% PAGE (sodium dodecyl sulfate-10\% polyacrylamide gel electrophoresis) western blotting (WB). $\beta$-Actin protein was used as loading control. Optical densitometry of selected bands were normalized relative to $\beta$-actin and plotted. (b) Interferon regulatory factor 3 (IRF3) protein bands and relative quantity to $\beta$-actin were shown with WB. Optical densitometry of IRF-3 bands were normalized relative to $\beta$-actin and indicated no significant difference between the groups. (c) Relative expression of IRF3-regulated gene, ISG15, was measured in HESN using quantitative reverse transcriptase-PCR. HESN expressed significantly lower levels of ISG15 compared with the HIV-negative (HIV-N) and HIV-P groups. $P$-values $<0.05$ are shown in the plots.
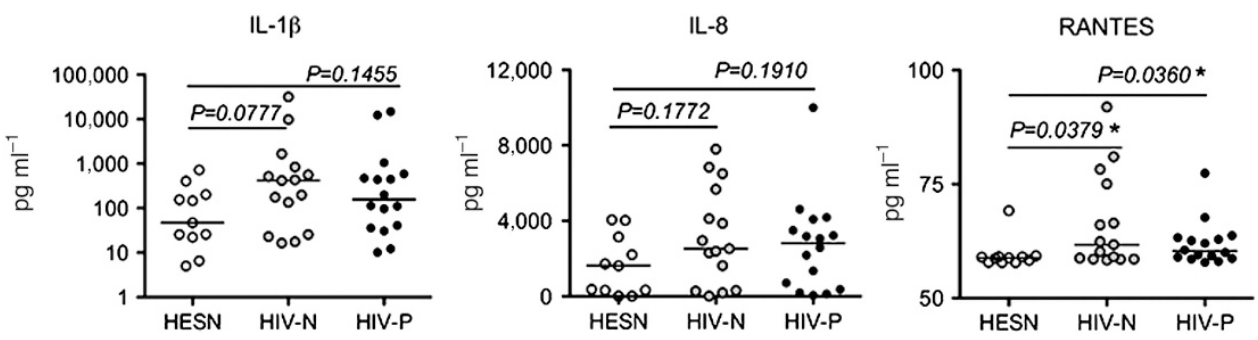

Figure 7 Reduced proinflammatory cytokines in cervicovaginal lavage (CVL) of HIV-resistant (HIV-R) commercial sex workers. Cytokines interleukin (IL)-1 $\beta$, IL-8 and RANTES (regulated and normal T cell expressed and secreted) were measured in CVL with enzyme-linked immunosorbent assay. RANTES was significantly reduced in HIV-R compared with the HIV-negative (HIV-N) and HIV-positive (HIV-P) groups, while IL-1 $\beta$ and IL-8 were slightly reduced in CVL from HIV-1-exposed seronegative (HESN). ${ }^{*} P<0.05$ and trend $P$-values $<0.2$ are shown in the plots. 


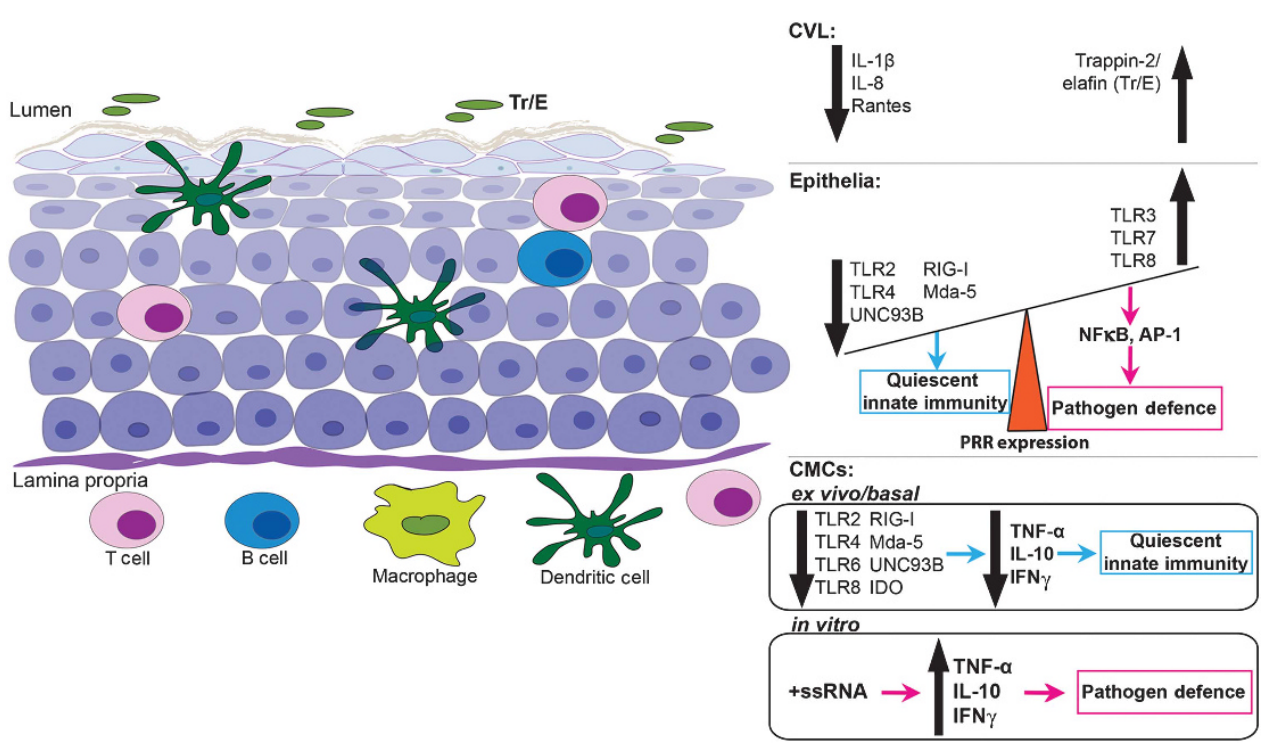

Figure 8 Proposed model and summary of factors contributing to local mucosal resistance to HIV-1 infection. Mucosa of the female genital tract consist of the lumen, epithelial cell layer, and lamina propria in which the main cells types include epithelial (cervical epithelial cells) and various mononuclear cells (cervical mononuclear cells (CMCs)), including T cells, B cells, macrophages, and dendritic cells. The genital mucosa is bathed in cervicovaginal lavage (CVL). Selected innate signaling proteins were downregulated in HIV-1-exposed seronegative (HESN) CMCs (black down arrow), generating low levels of cytokines to maintain quiescent innate immunity (blue arrows and rectangle). However, Toll-like receptor 7 (TLR7) and/or TLR8 were significantly stimulated by HIV-1 ssRNA, producing higher levels of cytokines to promote immune defense in HESN CMCs (pink arrows and rectangle). In light of our findings, we propose that the mucosal epithelium (light blue cells) limit HIV entry and infection by activation of endosomal nucleic acid-sensing TLRs (black up arrow) and innate transcription regulators, while other innate receptors are downregulated to reduce innate immune activation/inflammation (black down arrow). Total secreted proinflammatory cytokines in CVL are maintained at low levels (black down arrow), while innate defense and antiprotease factors, including trappin-2/elafin, are highly secreted in CVL, participating in pathogen defense. Thus, a delicate balance in the local innate genital mucosal microenvironment of HESN women serves to maintain an immune quiescent state reducing inflammation and immune activation (blue arrow and rectangle), while enhanced expression of endosomal TLRs provide rapid and potent activation of anti-viral defense (pink arrows and rectangle) in the HESN epithelium. AP-1, activator protein 1; IDO, indoleamine-2, 3-dioxygenase; IFN, interferon; IL, interleukin; Mda-5, melanoma-differentiation-associated gene 5; NF, nuclear factor; PRR, pattern-recognition receptor; RANTES, regulated and normal T cell expressed and secreted; RIG-I, retinoic acid-inducible gene-1; TNF, tumor necrosis factor.

CVL of HESN CSWs may serve to maintain genital mucosa integrity by protecting against inflammation, maintaining tight junctions and HIV-1 invasion.

\section{DISCUSSION}

Given that natural resistance to infection is primarily mediated by innate immune mechanisms and numerous studies suggesting that HESN women have novel immune responses in the cervicovaginal compartment associated with protection, the main objective of this study was to determine whether expression and function of innate sensing and signaling pathways were altered locally in the genital mucosa of HIV-1-resistant women in a well-characterized cohort of Kenyan CSWs. Our results demonstrate that CMCs and CECs isolated from HESN differentially expressed innate PRRs compared with HIV-1 positive and/or new negative susceptible CSWs. Notably, expression of numerous PRRs were significantly reduced in CMCs of HESN compared with HIV-P and/ or HIV-N. Importantly, TLR7 exhibited reduced expression over multiple years of assessment, despite more stringent criteria defining HIV-R over the years. These results are consistent with genomic analysis of blood and lymph nodes from naturally SIV-infected non-human primates (NHPs), namely sooty mangabeys, during chronic infection, which displayed significantly lower levels of innate immune activation, including PRRs RIG-I and Mda-5, compared with pathogenic SIV infection of rhesus macaques. ${ }^{33}$ Additionally, a more recent report of decreased immune activation determined by microarray analysis of gene expression signature patterns in whole blood of the same HESN cohort was demonstrated. ${ }^{12}$ UNC93B is essential for TLR3, TLR7, TLR8, and TLR9 signaling and function, as it is required for intracellular trafficking from the endoplasmic reticulum to endolysosomes in both mice and humans. ${ }^{24}$ Interestingly, significantly lower levels of UNC93B in CMCs of HESN women may effectively limit amounts of these TLRs in endosomes to, in turn, minimize signaling-induced cellular activation.

The established phenomenon of low levels of immune activation during the chronic phase of infection with high virus replication without progression to AIDS-like illness has been highlighted in recent years in SIV-infected natural hosts. ${ }^{34,35} \mathrm{~A}$ number of investigators have demonstrated that natural NHP hosts of SIV lack aberrant immune activation by at least five mechanisms, ${ }^{36}$ including: (1) Nef-mediated CD3 downmodulation to block the activation and apoptosis of infected T cells; (2) maintenance of intact mucosal barrier to prevent microbial translocation thereby reducing immune activation; (3) effective regulatory T-cell responses for anti-inflammation; (4) differential signaling and type I IFN production to avoid deleterious effects on T-cell function; and (5) reduced levels of CCR5 
expression on $\mathrm{CD}^{+}{ }^{+} \mathrm{T}$ cells. Natural NHP hosts for SIV seem to rely less on adaptive immune responses to control disease progression; however, they actively employ regulatory mechanisms to abort sustained immune activation due to associated detrimental effects. ${ }^{37}$ Thus, a key to lack of pathogenesis in naturally infected NHPs or resistance to infection appears to be related to decreased or controlled immune activation, which is consistent with the immune quiescence theory in HESN CSWs.

Our current evidence that unstimulated HESN CMCs expressed low levels of PRRs and reduced pro-inflammatory and anti-inflammatory cytokines in vitro is in sharp contrast with the significant response following in vitro stimulation with TLR7/TLR8 ligands. Maintenance of innate immune quiescence, especially in the genital tract, may be critically important for control of excessive immune activation and inflammation that would increase susceptibility to HIV-1 infection.

This evidence supports the currently accepted idea that immune activation is primarily disadvantageous to the host and a major driving force of HIV infection and pathogenesis. ${ }^{38}$ In addition, we determined that PRR-mediated signaling pathways are less activated in local immune cells in the genital mucosa of HESN CSWs, which potentially limits triggering activation of $\mathrm{CD} 4{ }^{+} \mathrm{T}$ cells, macrophages, and dendritic cells and, in turn, suppressing mucosal inflammation that enhances the risk of HIV transmission. Moreover, these cells have a greater capacity to highly functionally respond upon stimulation with viral ssRNA40. Our findings are partially consistent with a report that PBMCs from HESN individuals from another cohort showed significantly increased production of IL-6, TNF- $\alpha$, and IFN $\gamma$ following in vitro treatment with LPS and ssRNA40. ${ }^{39}$ In chronic HIV-1-infected patients immune activation occurs and concurrently drives the recruitment of new CD4 + T-cell targets for viral replication and elicits an immunosuppressive Treg response that blunts host defense against ongoing mucosal infection. ${ }^{23}$

Genital mucosal epithelium is a major portal of entry for HIV-1 sexual transmission. CECs provide a mechanical, chemical, and biological barrier to HIV-1 infection ${ }^{40}$ and are exposed to infectious and non-infectious HIV- $1 .{ }^{41}$ Here we report for the first time altered PRR expression patterns in human CECs from the three groups of CSWs. Similar to the observation in CMCs, CECs from HESN CSWs expressed significantly reduced levels of TLR2, TLR4, UNC93B, and Mda5 compared with HIV-P. However, unlike CMCs, CECs of HESN expressed significantly increased levels of TLR3 and TLR7 compared with the HIV-N and HIV-P groups. Furthermore, TLR3 and TLR7, which sense endolysosome dsRNA and ssRNA, were highly expressed in CECs of HESN in a correlative manner. This may reflect a mechanism of focused antiviral innate responses in genital ECs of HESN that is one of the keys to their protection against HIV. Previous reports showed that following HIV exposure in seronegative individuals, TLR7/8 engagement in blood induced enhanced innate and adaptive immune responses through robust release of immunological factors, which in turn interfere with HIV replication before viral integration. ${ }^{8}$ By contrast, two other dsRNA sensors, RIG-I and Mda-5, displayed reduced expression in CECs of HESN compared with HIV-P. The observation that expression of UNC93B was significantly decreased in CECs of HESN appears contradictory when comparing the increased expression levels of TLR3 and TLR7 in this group. This result may indicate that low amounts of UNC93B are sufficient to transport and initiate endosomal TLRs for signaling. Alternatively, it is possible that frequent exposure to HIV through sex work maintains elevated levels of these anti-virus-sensing TLRs in endosomal compartments, which depletes the endogenous levels of UNC93B in CECs. Indeed, it has been shown that HESN CSWs who take a break from sex work occasionally get infected following a return to sex work. ${ }^{42,43}$ These findings also raise the question whether continued exposure maintains altered levels of expression of innate PRRs in HESN or whether this innate phenotype is genetically determined.

Activation of innate immunity is a double-edged sword, as triggering of key transcription factors is an important step in PRR signaling cascade pathways and NF- $\kappa \mathrm{B}$ is required for HIV-1 gene expression via binding of the viral LTR. ${ }^{44,45}$ Therefore, it is not surprising that we observed activated NF- $\kappa B$ in CECs of HIV-P. Additionally, protein expression levels and activation of AP-1 exhibited the same pattern as NF- $\kappa B$. Our finding is consistent with reports of activation of NF- $\mathrm{KB}$ and AP- 1 in CD ${ }^{+}$T cells and U937 cells by viral Tat and Nef. ${ }^{46-48}$ Importantly, though, expression and activation of NF- $\mathrm{\kappa B}$ and AP-1 were also strongly increased in CECs of HESN compared with HIV-N. This may be due to higher levels of TLR3 and TLR7 in CECs of HESN. We speculate that the genital epithelium of HESN possesses innate mechanisms to eliminate invading HIV-1 before it can establish infectious replication. Highly expressed TLR3, TLR7, and downstream NF- $\kappa \mathrm{B}$ and AP-1 might be critically involved in innate antiviral responses in CECs of HESN.

In general, IRF3 is constitutively expressed, thus maintaining a constant level in the cytoplasm of cells. Strength and duration of IRF3 activity is regulated by post-translational modification, including phosphorylation, ubiquitination, ISGalytion, and S-glutathionylation. ${ }^{47}$ ISGalytion of IRF3 with ISG15 can stabilize the activated form of IRF3 and thereby enhance host innate antiviral responses. ${ }^{49}$ In our studies, IRF3 protein levels were observed to similar extents among the three groups, but we did not attempt to distinguish the forms of IRF3 here. Interestingly, one of the genes downstream of IRF3, expression of ISG15, was significantly decreased in HESN compared with the HIV-N and HIV-P groups. By contrast, HIV-P expressed elevated ISG15 which agrees with previous literature that indicated activated IRF3 in human monocytes treated with myristoylated recombinant HIV-1 Nef. ${ }^{50}$ However, the decreased expression of ISG15 most likely implies that IRF3 is not as active in HESN as in HIV-P CECs.

A recent study ${ }^{21}$ has shown that exposure of primary human genital epithelial cells in vitro to HIV gp120 altered tight junctions permitting virus to penetrate into the tissue. More 
recently, we demonstrated significantly increased HIV infection and integration, as well as increased proinflammatory cytokine production in cells expressing TLR2 (Henrick et al., submitted). Thus, lower expression of TLR2 in HESN CECs may provide a mechanism for decreased activation as well as reduced recruitment of HIV-susceptible cells.

Overall, our study reveals a local microenvironment of HIV resistance in the genital mucosa of HESN CSWs. This environment is characterized by significant differences in innate immune sensing and responsiveness locally in the genital mucosa of Kenyan HESN CSWs. Indeed, these women have multiple layers of innate defense against HIV in their genital tracts. Thus, there are barriers to HIV infection in the CVL, as well as at the level of genital ECs and mononuclear cells. In Figure 8, we have attempted to provide an overview and proposed model for local resistance to HIV-1 infection in the female genital mucosa reflecting the local resistant mucosal microenvironment. Our data indicate that expression of a number of PRRs was significantly reduced in CMCs of HESN women and basal cytokine secretion from HESN CMCs in vitro was significantly lower, yet greatly stimulated in the presence of ssRNA40. The same set of PRRs expressed at reduced levels in CMCs were expressed at low levels in CECs of HESN, but endosomal TLR3, TLR7, and TLR8 were expressed at correlatively and markedly elevated levels in HESN CECs, which may be a key contributor to resistance. Further, our results demonstrated that downstream transcription factors, NF- $\mathrm{KB}$ and AP-1, were strongly enhanced in both protein expression and activation in HESN CECs compared with HIVN. Finally, inflammatory cytokines, IL-1 $\beta$, IL-8, and RANTES, were expressed at lower levels in CVL of HESN. Our data partially supports the "immune quiescence" theory but extends to show heightened anti-HIV-specific responses in genital ECs and CMCs of HESN CSWs. Thus, endosomal viral RNA sensors in HESN genital epithelia and CMCs responded strongly when alerted to defense against HIV-1, while other PRRs maintained low expression, restricting mucosal inflammation, resulting in immune quiescence with a potent anti-viral knock-out punch. It still remains to be determined how expression of PRRs is differentially regulated in the epithelia of HESN. For example, whether these alterations are genetic or sustained by exposure to HIV-1 through sex work. Further understanding of the precise mechanisms of local PRR signaling and innate immunity in the genital mucosa of HESN individuals may provide clues for the development of novel prophylactic strategies to protect against sexual transmission of HIV-1 and inform HIV vaccine development.

\section{METHODS}

Ethics statement. This study was guided by the Helsinki Declaration on ethical principles for medical research involving human subjects. All studies were approved by University of Nairobi/Kenyatta National Hospital Ethical Review Board and the University of Manitoba Health Research Ethics Board. All patients provided written informed consent for collection of samples and subsequent analysis.
Human subjects. The women included in this study were comprised of CSWs who voluntarily enrolled themselves into the Pumwani Sex Worker cohort, as described elsewhere, ${ }^{1}$ and completed their 6-month re-evaluation between 26 June and 31 July, 2009. This observational cohort was established in 1985. The women enrolled in this cohort were re-evaluated for both sexual behavior as well as HIV seropositivity. During these re-evaluations, women were asked to complete a voluntary questionnaire regarding their duration in sex work, condom use, and number of sex clients per day. Despite behavioral counseling and condom promotion, it was estimated that these women have a yearly minimum of 64 unprotected sexual exposures. ${ }^{51}$ Additionally, women completed a physical examination in which endocervical swabs and blood were collected for testing. Additionally, a recent report from the same cohort indicated that no significant differences were reported for condom use, sexually transmitted infection, or demographic variables (including age and menopause) between HESN and negative controls from the same area. $^{52}$

Participants from the Pumwani CSWs cohort were enrolled in the June-August resurvey of 2009 for this study. Participants were divided into three groups: HESN, HIV-N, and HIV-P, based on their HIV seroconversion status at the time of collection and the years of commercial sex work as described in detail previously. ${ }^{20}$ Although 64 participants were initially enrolled, only 52 subjects had adequate yields of RNA valid for testing gene expression in CMCs and/or CECs. In this study, there were 43 CMC samples (HESN 11; HIV-N 16; HIV-P 16) and 44 CEC samples (12 HESN, 16 HIV-N, and 16 HIV-P) evaluated.

Isolation of mucosal samples. The following genital tract specimens were collected:

1. CVL was collected by flushing the ectocervix with $2 \mathrm{ml}$ of sterile phosphate-buffered saline (PBS) and collecting fluid from the posterior fornix region. Samples were kept cool for transport and frozen at $-80^{\circ} \mathrm{C}$ after removal of cellular fraction.

2. CMC: First the cervix was scraped to remove freshly sloughed off cells and placed in $10 \mathrm{ml}$ PBS in a Facol tube. Next, the endocervical cytobrush was inserted into the endocervical os, rotated $360^{\circ}$, immediately placed into $1 \mathrm{~mL}$ PBS, and added to the same tube. The samples were then briefly vortexed for 20-30 s to loosen the cells and $10 \mathrm{ml}$ of RPMI was added. Later, cells were separated using a Ficoll gradient in which cells that remained suspended on the gradient were considered CMCs, while pelleted cells were considered CECs. CMCs were resuspended in $1 \mathrm{~mL}$ RPMI, counted, and separated for use in cell culture assays or mRNA analysis after TRIzol treatment.

3. CECs: Following Ficoll gradient separation, CECs were washed with $12 \mathrm{ml}$ of cold PBS and centrifuged at 1,700 r.p.m. for $10 \mathrm{~min}$ and dissolved in $0.8 \mathrm{ml}$ of TRIzol. Purity of CECs were assessed by measuring T- and B-cell markers, CD3 and CD19, respectively. A ribosomal protein gene, $\mathrm{L} 32$, was used to normalize quantification. PBMC and a vaginal epithelial cell line, VK2, were used as positive and negative controls for cellular contamination, respectively. Based on these comparisons, we estimate approximately $90 \%$ of the cells in the preparation are CECs.

Qunatitative reverse transcriptase-PCR. RNA extraction and quantitative reverse transcriptase-PCR are described previously. Briefly, total RNA was extracted from the CMC and CE samples in TRIzol as per the manufacturer's instructions (Invitrogen, Burlington, ON, Canada), followed by DNA-free treatment (Ambion, Burlington, ON, Canada). In all, $0.5 \mu \mathrm{g}$ RNA was converted into cDNA with Superscript reverse transcriptase III (Invitrogen) in $40 \mu \mathrm{l}$ of reaction 
mixture. Real-time quantitative PCR was performed in $25 \mu$ l volume containing $6 \mathrm{ng}$ cDNA with TaqMan or SYBR Green (Applied Biosystems, Burlington, ON, Canada). 18S rRNA was quantified and used for normalizing all of gene expression quantity. The oligouncleotide primers and probes labeled with $6 \mathrm{FAM}$ at $5^{\prime}$-end and a nonflorescent quencher at $3^{\prime}$-end are listed in Supplementary Table S1 online. PCR was run with the standard program: at $95^{\circ} \mathrm{C}$ for $10 \mathrm{~min}, 40$ times of cycling at $95^{\circ} \mathrm{C}$ for $15 \mathrm{~s}$, and at $60^{\circ} \mathrm{C}$ for $1 \mathrm{~min}$ in duplicate in a 96-well plate with a Sequencing Detection System 7900HT (Applied Systems, Burlington, ON, Canada).

In vitro stimulation of CMCs and response to pathogen-associated molecular patterns. Freshly isolated CMCs were suspended in HyQ RPMI 1640 (Invitrogen), 0.5\% penicillin and amphotericin B, and 10\% fetal bovine serum (Invitrogen) and then incubated in a 96-well round-bottomed culture plate $\left(10^{5} \mathrm{CMCs}\right.$ per $200 \mathrm{ul}$ per well $)$ at $5 \%$ $\mathrm{CO}_{2}$ at $37^{\circ} \mathrm{C}$. TLR agonists were added to the $\mathrm{CMC}$ cell suspensions as follows: $0.01 \mu \mathrm{g} \mathrm{ml}^{-1}$ LPS (Invivogen, Cedarlane, Burlington, ON, Canada), $1 \mathrm{~mm}$ ssRNA40/LyoVec40 (Invivogen), $1 \mathrm{~mm}$ imiquimod (Invivogen), or untreated complete RPMI 1640 media, and cultured overnight. The harvested supernatants were assayed for cytokines (IL-2, IL-4, IL-6, IL-10, TNF- $\alpha$, and IFN $\gamma$ ) using cytometric bead array (BD Biosystems, Mississauga, ON, Canada) on a FACScan flow cytometer (BD, Mississauga, ON, Canada), and the analysis was completed using BD CBA Software (BD Biosciences, Mississauga, ON, Canada).

Western blot. Protein isolation was completed after RNA extraction from TRIzol-stored CEC, as per the manufacturer's instruction (Invitrogen). Protein was dissolved in $1 \%$ sodium dodecyl sulfate (SDS) and quantified with dendritic cell Protein Assay (Bio-Rad, Mississauga, ON, Canada). In all, $10 \mu \mathrm{g}$ protein samples were loaded on SDS-PAGE (polyacrylamide gel electrophoresis) and transferred to PVDF membrane. The membranes were individually bound with antip65 (Santa Cruz, Santa Cruz, CA), IRF3 (IBL, Takasakishi, Gunma, Japan), pho I $\kappa \beta \alpha$, c-Jun, pho c-Jun, and $\beta$-actin (Cell Signaling, New England Biolabs Ltd., Whitby, ON, Canada) primary antibodies. Secondary antibodies included anti-rabbit immunoglobulin G-HRP (Bio-Rad) or anti-mouse immunoglobulin G-horseradish peroxidase (Santa Cruz). Membranes were developed with Super Signal West Pico or Femto Chemiluminescent Substrates (Thermo, Burlington, ON, Canada) and exposed to CL-X films. Protein band density was quantified by scanning and analyzed with ImageJ (http://imagej.en. softonic.com).

Enzyme-linked immunosorbent assay. Cytokine levels, including IL-1 $\beta$, IL-8, and RANTES (CCL5), were determined using DouSet ELISA as per the manufacturer's instructions (R\&D, Minneapolis, MN).

Statistics. All data were analyzed with Mann-Whitney $t$-test between the two groups, and two-tailed $P$-values were calculated using the GraphPad Prism, version 4 (San Diego, CA).

SUPPLEMENTARY MATERIAL is linked to the online version of the paper at http://www.nature.com/mi

\section{ACKNOWLEDGEMENTS}

We thank the volunteers of the Pumwani sex worker cohort for their invaluable time and contribution to this project. We sincerely acknowledge support of the clinic staff, including Dr Charles Wachihi and Dr Walter Jaoko of the Department of Medical Microbiology at the University of Nairobi, Nairobi, Kenya and laboratory technicians of the University of Nairobi for assistance with sample collection and collaboration. We are also grateful for the supervision provided by Dr Makobu Kimani (Department of Medical Microbiology, University of Nairobi) and Dr Solomon Mpoke (Kenya Medical Research Institute, Nairobi, Kenya). This study was funded by grants from the Bill and Melinda Gates Foundation and the Canadian Institutes of Health Research (CIHR). The funders had no role in study design, data collection and analysis, decision to publish, or preparation of the manuscript.

\section{DISCLOSURE}

The authors declared no conflict of interest.

(c) 2014 Society for Mucosal Immunology

\section{REFERENCES}

1. Fowke, K.R. et al. Resistance to HIV-1 infection among persistently seronegative prostitutes in Nairobi, Kenya. Lancet 348, 1347-1351 (1996).

2. Were, W.A. et al. Undiagnosed HIV infection and couple HIV discordance among household members of HIV-infected people receiving antiretroviral therapy in Uganda. J. Acquir. Immune Defic. Syndr. 43, 91-95 (2006).

3. Tomescu, C., Abdulhaqq, S. \& Montaner, L.J. Evidence for the innate immune response as a correlate of protection in human immunodeficiency virus (HIV)-1 highly exposed seronegative subjects (HESN). Clin. Exp. Immunol. 164, 158-169 (2011).

4. Clerici, M. et al. T-lymphocyte maturation abnormalities in uninfected newborns and children with vertical exposure to HIV. Blood 96, 3866-3871 (2000).

5. Vigano, A. et al. Long-term resistance to HIV infection in vertical HIV infection: cytokine production, HIV isolation, and HIV phenotype define long-term resistant hosts. Pathobiology 65, 169-176 (1997).

6. Kaul, R. et al. HIV-1-specific mucosal IgA in a cohort of HIV-1-resistant Kenyan sex workers. AIDS 13, 23-29 (1999).

7. Kaul, R. et al. HIV-1-specific mucosal CD8 + lymphocyte responses in the cervix of HIV-1-resistant prostitutes in Nairobi. J. Immunol. 164, 1602-1611 (2000).

8. Kaul, R. et al. Late seroconversion in HIV-resistant Nairobi prostitutes despite pre-existing HIV-specific CD8 + responses. J. Clin. Invest. 107, 341-349 (2001).

9. Iqbal, S.M. et al. Elevated T cell counts and RANTES expression in the genital mucosa of HIV-1-resistant Kenyan commercial sex workers. J. Infect. Dis. 192, 728-738 (2005).

10. Card, C.M. et al. Decreased immune activation in resistance to HIV-1 infection is associated with an elevated frequency of CD4 $(+)$ CD25 $(+)$ FOXP3 $(+)$ regulatory T cells. J. Infect. Dis. 199, 1318-1322 (2009).

11. McLaren, P.J. et al. HIV-exposed seronegative commercial sex workers show a quiescent phenotype in the CD4 $+\mathrm{T}$ cell compartment and reduced expression of HIV-dependent host factors. J. Infect. Dis. 202 (Suppl 3), S339-S344 (2010).

12. Songok, E.M. et al. Microarray analysis of HIV resistant female sex workers reveal a gene expression signature pattern reminiscent of a lowered immune activation state. PloS One 7, e30048 (2012).

13. Iqbal, S.M. et al. Elevated elafin/trappin-2 in the female genital tract is associated with protection against HIV acquisition. AIDS 23, 1669-1677 (2009).

14. Burgener, A. et al. Comprehensive proteomic study identifies serpin and cystatin antiproteases as novel correlates of HIV-1 resistance in the cervicovaginal mucosa of female sex workers. J. Proteome Res. 10, 5139-5149 (2011).

15. Schweneker, M., Favre, D., Martin, J.N., Deeks, S.G. \& McCune, J.M. HIV-induced changes in T cell signaling pathways. J. Immunol. 180, 6490-6500 (2008)

16. Bafica, A., Scanga, C.A., Schito, M., Chaussabel, D. \& Sher, A. Influence of coinfecting pathogens on HIV expression: evidence for a role of Toll-like receptors. J. Immunol. 172, 7229-7234 (2004).

17. Baenziger, S. et al. Triggering TLR7 in mice induces immune activation and lymphoid system disruption, resembling HIV-mediated pathology. Blood 113, 377-388 (2009).

18. Sachdeva, M., Fischl, M.A., Pahwa, R., Sachdeva, N. \& Pahwa, S. Immune exhaustion occurs concomitantly with immune activation and decrease in regulatory T cells in viremic chronically HIV-1-infected patients. J. Acquir. Immune Defic. Syndr. 54, 447-454 (2010).

19. Meier, A. et al. MyD88-dependent immune activation mediated by human immunodeficiency virus type 1-encoded Toll-like receptor ligands. J. Virol. 81, 8180-8191 (2007). 
20. Lester, R.T. et al. Toll-like receptor expression and responsiveness are increased in viraemic HIV-1 infection. AIDS 22, 685-694 (2008).

21. Drannik, A.G. et al. Anti-HIV-1 activity of elafin is more potent than its precursor's, trappin-2, in genital epithelial cells. J. Virol. 86, 4599-4610.

22. Trivedi, H.N. et al. Resistance to HIV-1 infection among African sex workers is associated with global hyporesponsiveness in interleukin 4 production. FASEB J. 15, 1795-1797 (2001).

23. Haase, A.T. Perils at mucosal front lines for HIV and SIV and their hosts. Nat. Rev. Immunol. 5, 783-792 (2005).

24. Saitoh, S. \& Miyake, K. Regulatory molecules required for nucleotidesensing Toll-like receptors. Immunol. Rev. 227, 32-43 (2009).

25. Gringhuis, S.I. et al. HIV-1 exploits innate signaling by TLR8 and DC-SIGN for productive infection of dendritic cells. Nat. Immunol. 11, 419-426 (2010).

26. Schellenberg, J.J. \& Plummer, F.A. The microbiological context of HIV resistance: vaginal microbiota and mucosal inflammation at the viral point of entry. Int. J. Inflamm. 2012, 131243 (2012).

27. Fallarino, F. \& Grohmann, U. Using an ancient tool for igniting and propagating immune tolerance: IDO as an inducer and amplifier of regulatory T cell functions. Curr. Med. Chem. 18, 2215-2221 (2011).

28. Favre, D. et al. Tryptophan catabolism by indoleamine 2,3-dioxygenase 1 alters the balance of $\mathrm{TH} 17$ to regulatory T cells in HIV disease. Sci. Transl. Med. 2, 32 ra36 (2010).

29. Favre, D. et al. Critical loss of the balance between Th17 and T regulatory cell populations in pathogenic SIV infection. PLoS Pathogens 5, e1000295 (2009).

30. Beg, A.A., Finco, T.S., Nantermet, P.V. \& Baldwin, A.S. Jr. Tumor necrosis factor and interleukin-1 lead to phosphorylation and loss of I kappa B alpha: a mechanism for NF-kappa B activation. Mol. Cell. Biol. 13, 3301-3310 (1993).

31. Brockman, J.A. et al. Coupling of a signal response domain in I kappa B alpha to multiple pathways for NF-kappa B activation. Mol. Cell. Biol. 15, 2809-2818 (1995).

32. Brown, K., Gerstberger, S., Carlson, L., Franzoso, G. \& Siebenlist, U. Control of I kappa B-alpha proteolysis by site-specific, signal-induced phosphorylation. Science 267, 1485-1488 (1995).

33. Bosinger, S.E. et al. Global genomic analysis reveals rapid control of a robust innate response in SIV-infected sooty mangabeys. J. Clin. Invest. 119, 3556-3572 (2009).

34. Silvestri, G. et al. Nonpathogenic SIV infection of sooty mangabeys is characterized by limited bystander immunopathology despite chronic high-level viremia. Immunity 18, 441-452 (2003).

35. Beaumier, C.M. et al. CD4 downregulation by memory CD4 + Tcells in vivo renders African green monkeys resistant to progressive SIVagm infection. Nat. Med. 15, 879-885 (2009).

36. Kirchhoff, F. Is the high virulence of HIV-1 an unfortunate coincidence of primate lentiviral evolution?. Nat. Rev. Microbiol. 7, 467-476 (2009).

37. Manches, O. \& Bhardwaj, N. Resolution of immune activation defines nonpathogenic SIV infection. J. Clin. Invest. 119, 3512-3515 (2009).
38. Mogensen, T.H., Melchjorsen, J., Larsen, C.S. \& Paludan, S.R. Innate immune recognition and activation during HIV infection. Retrovirology 7, 54 (2010).

39. Biasin, M. et al. TLR activation pathways in HIV-1-exposed seronegative individuals. J. Immunol. 184, 2710-2717 (2010).

40. Kaushic, C., Ferreira, V.H., Kafka, J.K. \& Nazli, A. HIV infection in the female genital tract: discrete influence of the local mucosal microenvironment. Am. J. Reprod. Immunol. 63, 566-575 (2010).

41. Howell, A.L. et al. Human immunodeficiency virus type 1 infection of cells and tissues from the upper and lower human female reproductive tract. J. Virol. 71, 3498-3506 (1997).

42. Swingler, S., Easton, A. \& Morris, A. Cytokine augmentation of HIV-1 LTRdriven gene expression in neural cells. AIDS Res. Hum. Retroviruses $\mathbf{8}$, 487-493 (1992).

43. Borellini, F. et al. Increased DNA binding and transcriptional activity associated with transcription factor Sp1 in K562 cells transfected with the myeloid-specific c-fes tyrosine kinase gene. J. Biol. Chem. 266, 15850-15854 (1991).

44. Varin, A. et al. Exogenous Nef protein activates NF-kappa B, AP-1, and C-Jun N-terminal kinase and stimulates HIV transcription in promonocytic cells. Role in AIDS pathogenesis. J. Biol. Chem. 278, 2219-2227 (2003).

45. Fortin, J.F., Barat, C., Beausejour, Y., Barbeau, B. \& Tremblay, M.J. Hyperresponsiveness to stimulation of human immunodeficiency virus-infected $\mathrm{CD} 4+\mathrm{T}$ cells requires Nef and Tat virus gene products and results from higher NFAT, NF-kappaB, and AP-1 induction. J. Biol. Chem. 279, 39520-39531 (2004).

46. Bibeau-Poirier, A. et al. Involvement of the IkappaB kinase (IKK)-related kinases tank-binding kinase $1 / 1 \mathrm{KKi}$ and cullin-based ubiquitin ligases in IFN regulatory factor-3 degradation. J. Immunol. 177, 5059-5067 (2006).

47. Lu, G. et al. ISG15 enhances the innate antiviral response by inhibition of IRF-3 degradation. Cell. Mol. Biol. (Noisy-le-grand) 52, $29-41$ (2006).

48. Prinarakis, E., Chantzoura, E., Thanos, D. \& Spyrou, G. S-glutathionylation of IRF3 regulates IRF3-CBP interaction and activation of the IFN beta pathway. EMBO J. 27, 865-875 (2008).

49. Mangino, G. et al. In vitro treatment of human monocytes/macrophages with myristoylated recombinant Nef of human immunodeficiency virus type 1 leads to the activation of mitogen-activated protein kinases, IkappaB kinases, and interferon regulatory factor 3 and to the release of beta interferon. J. Virol. 81, 2777-2791 (2007).

50. Nazli, A. et al. Exposure to HIV-1 directly impairs mucosal epithelial barrier integrity allowing microbial translocation. PLoS Pathogens 6, e1000852 (2010).

51. Horton, R.E. et al. Cervical HIV-specific IgA in a population of commercial sex workers correlates with repeated exposure but not resistance to HIV. AIDS Res. Hum. Retroviruses 25, 83-92 (2009).

52. Chege, D. et al. Blunted IL17/IL22 and pro-inflammatory cytokine responses in the genital tract and blood of HIV-exposed, seronegative female sex workers in Kenya. PloS One 7, e43670 (2012). 